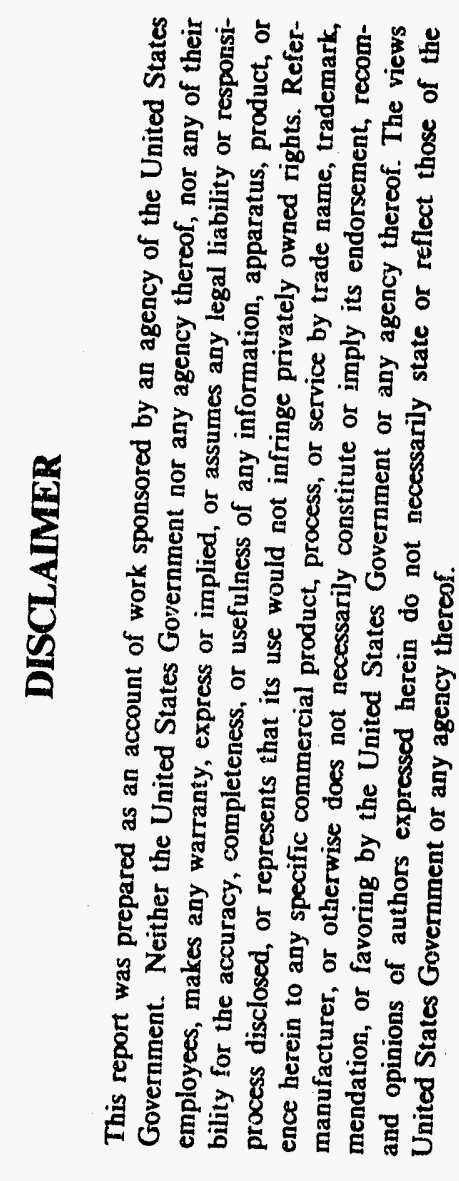

$\mathrm{DOE} / \mathrm{ER} / 60684--\mathrm{T} 1$

DE92 011124

\title{
FLUORESCENCE-DETECTED DNA SEQUENCING
}

FINAL TECHNICAL REPORT

Grant No. DE-FG06-88ER60684

For The Period September 30, 1988 - September 29, 1990

by

Richard P. Haugland, Principal Investigator

Molecular Probes, Inc.

4849 Pitchford Avenue

Eugene, Oregon 97402 


\title{
Molecular Probes, Inc. \\ 4849 PITCHFORD AVE. • P.O. BOX 22010 • EUGENE, OR 97402 \\ (503)344-3007 • TELEX 858721 MOLECULAR • FAX (503)344-6504
}

\author{
Final Progress Report \\ Research Grant No. DE-FG06-88ER-60684 \\ Richard P. Haugland, Principal Investigator \\ Hee Chol Kang, Project Director
}

Our research effort funded by this grant primarily focused on development of suitable fluorescent dyes for DNA sequencing studies. Prior to our efforts, the dyes being used in cormmercial DNA sequencers were various versions of fluorescein dyes for the shorter wavelengths and of rhodamine dyes for the longer wavelengths. Our initial goal was to synthesize a set of four dyes that could all be excited by the 488 and $514 \mathrm{~nm}$ line of the argon laser lines and that have emission spectra that minimize spectral overlap. The specific result sought was higher fluorescent intensity, particularly of the longest wavelength dyes than was available using existing dyes. Another important property of the desired set of dyes was uniform ionic charge in order to have minimum interference on the electrophoretic mobility during the sequencing.

During the period of this grant and in subsequent research, we prepared and characterized four types of dyes: fluorescent bifluorophores, derivatives of rhodamine dyes, derivatives of rhodol dyes and cerivatives of boron dipyrromethene difluoride (BODIPY ${ }^{\mathrm{TM}}$ ) dyes. We were quite successful in three of the four areas and these dyes are now being used or evaluated by several companies for fluorescence-detected DNA sequencing, fluorescent in situ hybridization assays and as labels for various immunoassays. Changes in the technology that have occurred, particularly in the use of multiple lasers that have output at longer wavelengths (especially $543,568,590$, and $633 \mathrm{~nm}$ ) have made the fluorophores that we developed even more valuable for these applications.

\section{Fluorescent Bifluorophores}

Our primary proposal was to use the support of this grant to develop a set of dyes that we termed "bifluorophores." We designed, synthesized and evaluated a number of bifluorophores, in which one fluorescent molecule ("donor") was covalently attached to a second longer-wavele.ngth fluorophore ("acceptor") so that the energy absorbed by the donor is transferred (in the excited state) to the acceptor dye, resulting in emission at the (longer wavelength) acceptor emission maximum. This wavelength shifting should have the net result of a higher absorbance at the exciting wavelength and a higher emission intensity at a wavelength that is significantly Stokes shifted from the excitation wavelength. 
Most of our research focused on derivatives of fluorescein and tetramethylrhodamine as the donor and acceptor molecules, respectively, since these dyes were already being used in DNA sequencing, they have reasonable good match of spectral properties and we already had easy availability of several versions of their reactive forms from which to prepare bifluorophores. We synthesized several sets of fluorescein-tetramethylrhodamine bifluorophores in an attempt to get optimal spectral characteristics. Typical examples prepared include those in Figure 1.

The important factors in our synthetic strategy to develop appropriate fluoresceintetramethylrhodamine bifluorophores included the distance between fluorescein and tetramethylrhodamine molecules, the position of attachment of the two fluorophores and the nature of chemical linkers between the donor and the acceptor molecules. We varied these in several ways as shown by the compounds drawn.

Among the above bifluorophores, Compound 1 was shown to have the best spectral properties. The absorption spectrum of Compound 1 shows the sum of absorption (Figure $2 \mathrm{a}$ ) from the two fluorophores and the fluorescence (Figure 2b) is observed at the emission maximum of tetramethylrhodamine at $575 \mathrm{~nm}$ when the compound is excited at $488 \mathrm{~nm}$ with minimal residual fluorescence of the donor at about $520 \mathrm{~nm}$ in pH 7.0 phosphate saline buffer solution. This confirms the almost quantitative energy transfer with a Stokes shift of about $90 \mathrm{~nm}$ and the potential feasibility of the approach. This value is considerably higher than can be obtained with any other single fluorophore known in this wavelength region. When fluorescence of bifluorophore 1 excited at 488 $\mathrm{nm}$ is compared with that of tetramethylrhodamine acceptor alone excited at $488 \mathrm{~nm}$, the fluorescence is about four times as great in the bifluorophore 1.

We prepared the amine-reactive succinimidyl ester from bifluorophore 1 (Compound 12, Figure 4). The bifluorophore 12 was conjugated with oligonucleotides and several proteins. The resulting conjugates still have the property of being excitable with an argon laser line at $488 \mathrm{~nm}$ with emission being monitored at an unisually long wavelength.

In order to get an even higher Stokes shift, we prepared several fluorescein-x-rhodamine bifluorophores. In one example, Compound 9, (Figure 3) fluorescence was observed at about $600 \mathrm{~nm}$ when excited at $488 \mathrm{~nm}$ with about $2 \%$ residual fluorescence of the fluorescein donor molecule.

Furthermore, we prepared and characterized a fluorescein resorufin bifluorophore 10 and BODIPY-tetramethylrhodamine bifluorophore 11 (Figure 3) to determine what the charge interaction between the donor and the acceptor would do for the energy transfer.

Overall, however, our results with bifluorophore approach were disappointing. The energy transfer was inconsistent and unpredictable. In the majority of cases we did not observe quantitative transfer, even when the probes were close, had excellent spectral overlap and had relatively free rotation about bonds - exactly the conditions where the theory would predict quantitative transfer. We attempted to resolve these problems by introducing relatively rigid spacers that would prevent ground state interaction of the two fluorophores, including a short polyproline spacer, but these resulted were not successful. The second problem that we found was that the relatively low quantum yield of the longer wavelength rhodamine dyes limits the ultimate amount of fluorescence that could be obtained, even if quantitative transfer could be obtained. 
Because of the problems with the bifluorophore fluorescence yields, we turned to the improvement of other types of dyes that were originally discovered during an NIH-sponsored Small Business Innovation Research grant. Some of these have developed into excellent candidates for DNA sequencing and other applications and their commercial application is about to commence.

\section{Derivatives of Rhodamine Dyes}

We have successfully developed a set of four rhodamine dyes which have reasonably good spectral properties for DNA sequencing. Their structures and spectral data are shown in Figure 5. These dyes have a reactive succinimidyl ester group, so that they can be covalently attached to amine terminated oligonucleotides, nucleic acids and other proteins. Since these series of dyes have the same ionic charge, they have very little interference with electrophoretic separations during the sequencing. A company has been using some of these successfully in their commercial DNA sequencing system. Some of these dyes have recently found use in commercial in situ hybridization applications.

\section{Derivatives of Rhodol Dyes}

We have developed a new class of reactive dyes that we called "rhodol" dyes. Some typical examples and their spectral data are shown in Figure 6. These dyes are intermediate between fluorescein and rhodamine derivatives in structure and spectral properties. They show better photostability than fluorescein derivatives, low $\mathrm{pH}$ sensitivity and higher fluorescent quantum yields in aqueous solution than dyes such as tetramethylrhodamine. Many reactive forms of these rhodol dyes were prepared and utilized for conjugation with oligonucleotides, dextrans and several proteins. In this project, at least sixty (60) new compounds were prepared and a U.S. Patent Application $07 / 509,360$ was filed for chemically reactive rhodol dyes in which this grant has been given certain rights. The limitation of this group of dyes for multi-color DNA sequencing has been the difficulty in shifting the spectra to wavelengths past $\sim 560 \mathrm{~nm}$.

\section{Derivatives of Boron Dipyrromethene Difluoride (BODIPY) Dyes}

The boron dipyrromethene difluoride ("BODIPY") that we developed with the support of a Phase I NIH SBIR grant, continued to develop with support of this DOE grant and, following termination of the DOE grant, have further improved with support of the second phase NIH SBIR grant and with company funds have become probably the best set of dyes for DNA sequencing applications, particularly since companies have started to use the longer wavelength excitation sources. Through agreements with at least four other companies, we are starting to move into commercial applications of these dyes in sequencing and other applications.

The "original" BODIPY dye had fluorescein-like spectra with a much narrower band width for both excitation and emission spectra than fluorescein, which makes it of significant utility for DNA sequencing. The work funded by this DOE-sponsored grant concentrated on developing dyes that could be better excited by the 488 and 514 Ar laser lines. The fast rise and fall of the spectra of this class of dyes makes an exact match particularly important. Some of the best dyes that we found are given in Figure 7. Compound 23 has been prepared as a rhodamine $6 \mathrm{G}$ substitute during this research grant period. We have evaluated its properties fully and found several advantages over rhodamine $6 \mathrm{G}$. 
Subsequent to the work on this grant, we have been able to develop longer wavelength BODIPY derivatives as rhodamine substitutes. The advantages of the BODIPY dyes are their narrow excitation and emission bandwidths, their higher fluorescent quantum yield even in aqueous solution and their relatively high photostability. We have now been able to develop a number of longer wavelength BODIPY derivatives with emission maxima up to $700 \mathrm{~nm}$.

We are just about to commercialize modified oligonucleotides that contain some of the BODIPY and other dyes for research applications. Although we did not get to use these dyes directly in sequencing, research at the companies that have evaluated them for this purpose has been quite successful and our ability to tailor these BODIPY dyes to almost exactly match excitation or emission wavelength requirements has made these probably the best dyes for DNA sequencing available.

In summary, a large number of fluorescent dyes have been synthesized and their spectral properties evaluated. Through support of this grant, other grants and company funds we have made several improvements in dyes that will have broad utility in the Human Genome Initiative.

Sincerely,

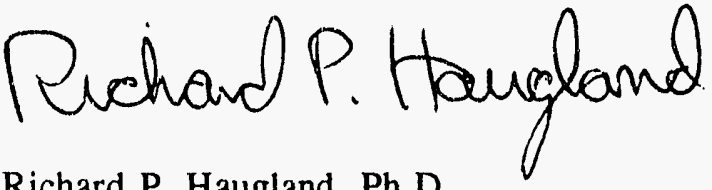

Richard P. Haugland, Ph.D.

President 

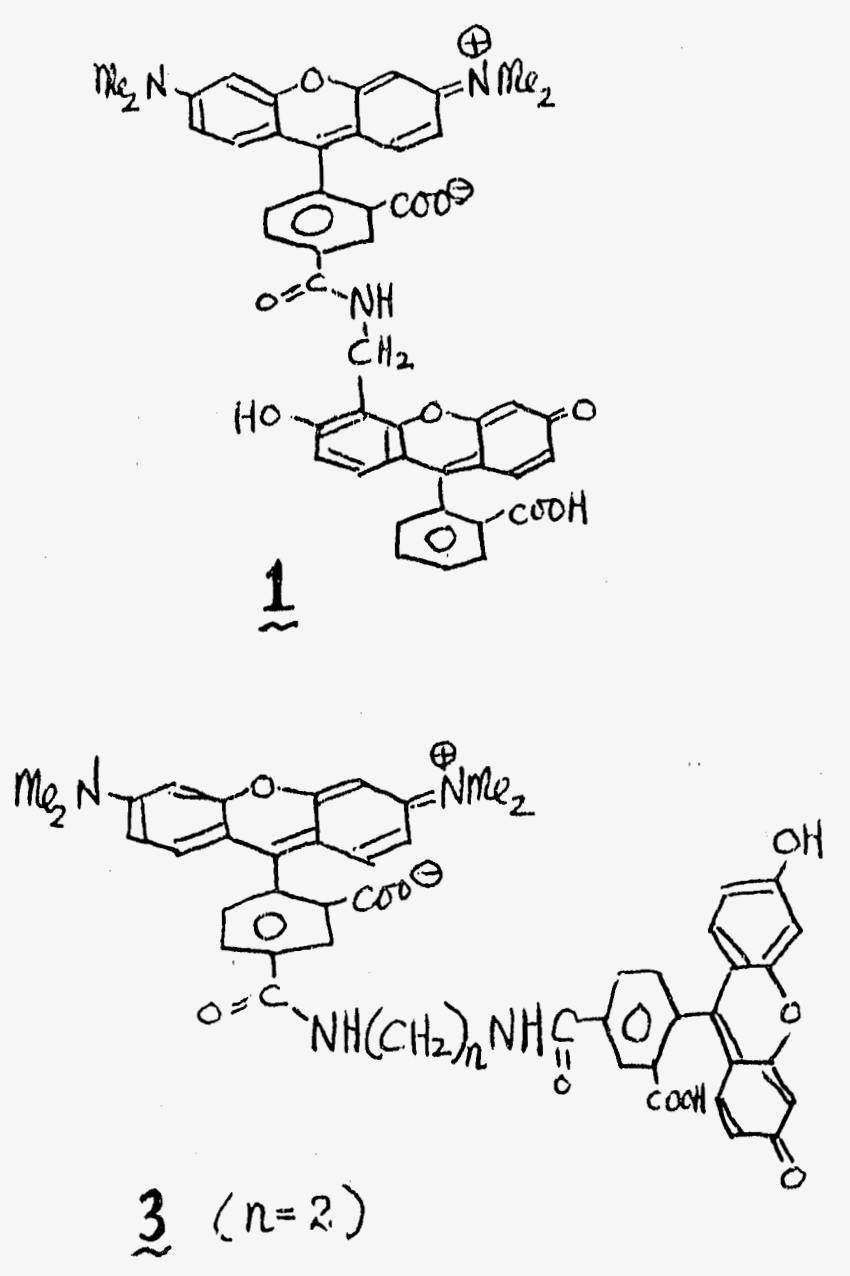

$4(n=5)$

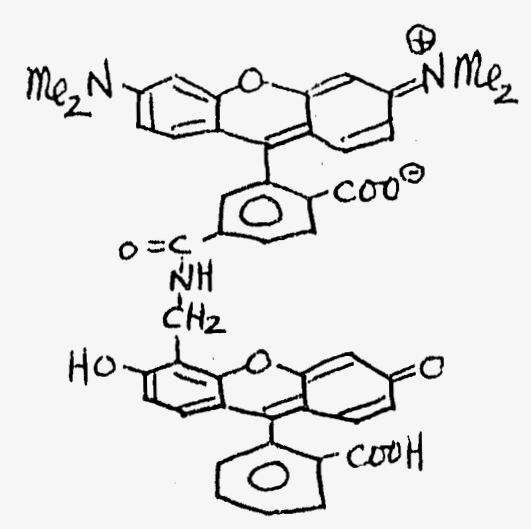

2

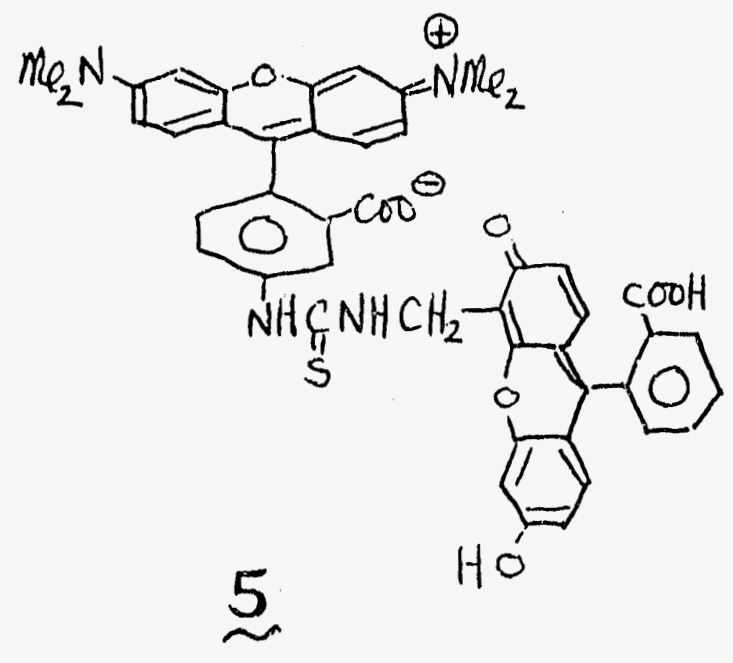

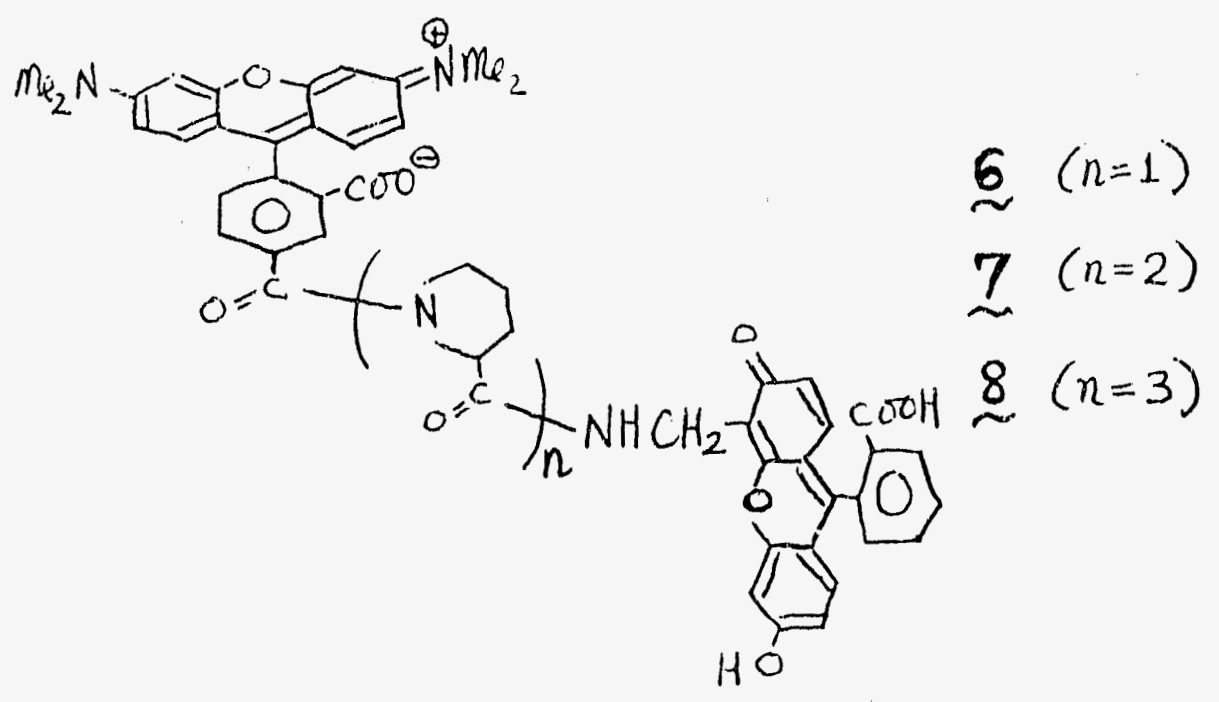

Figure 1 


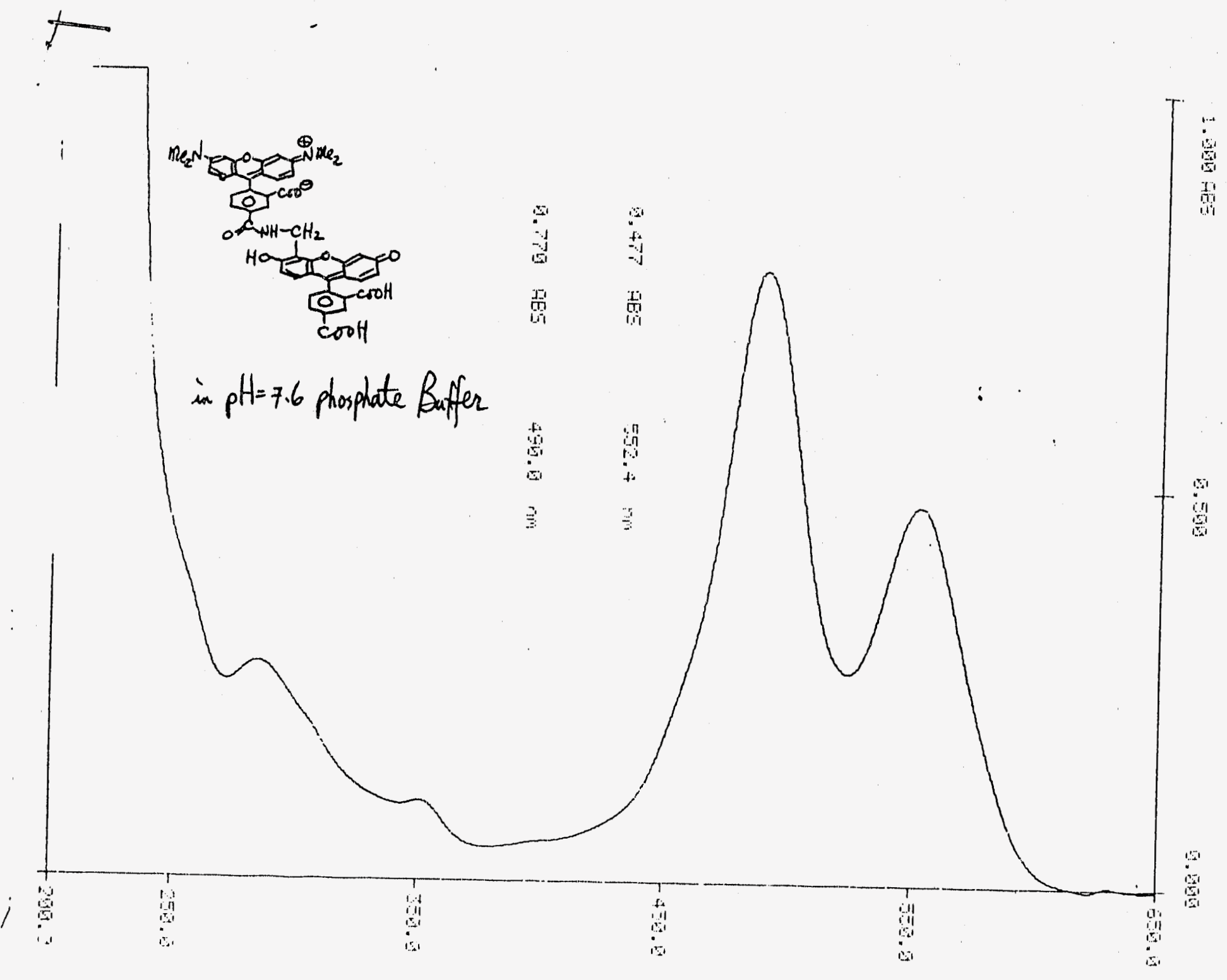

Figure aa: Absorption Spectrum of Compound $\mathbf{1}$ 


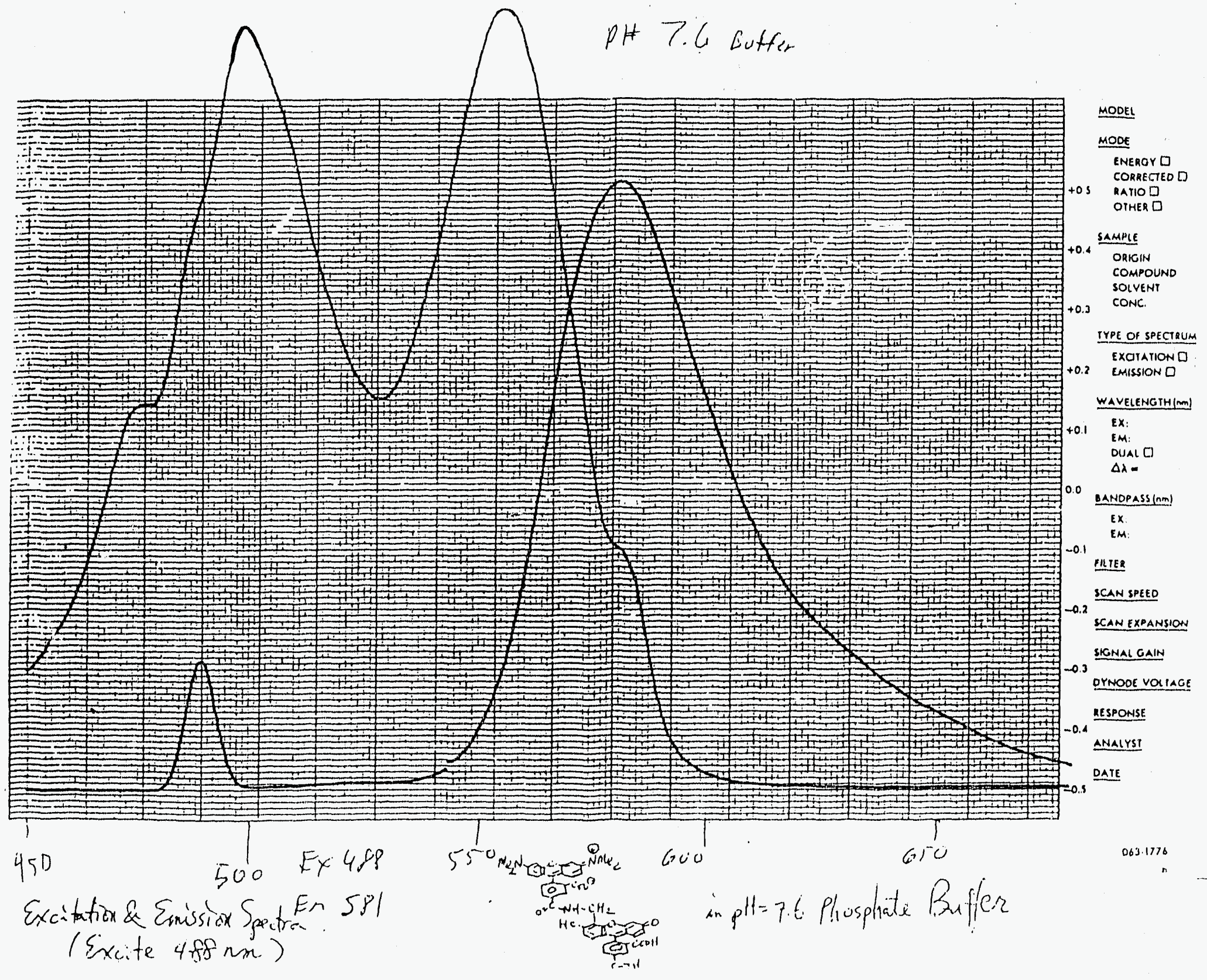

Figure 2b: Excitation and Emission Spectra of Compound 1 

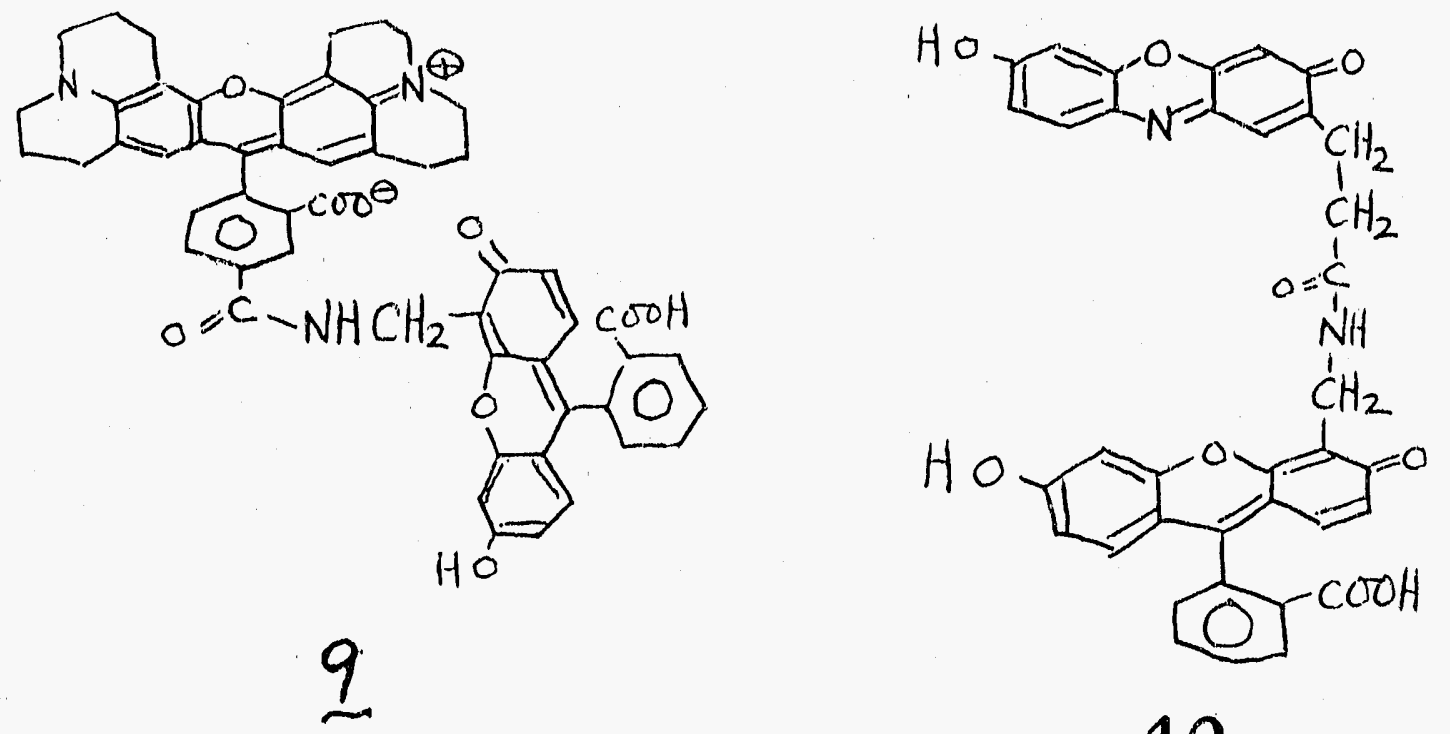

10

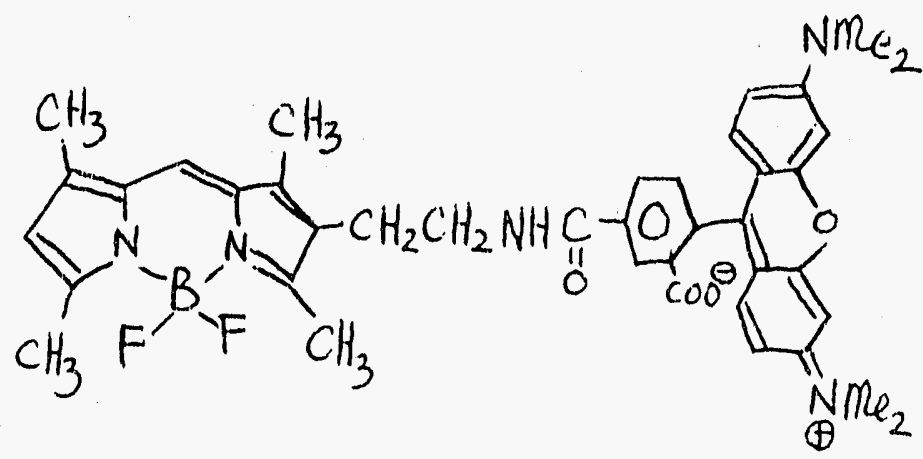

$\stackrel{11}{\sim}$

Figure 3 


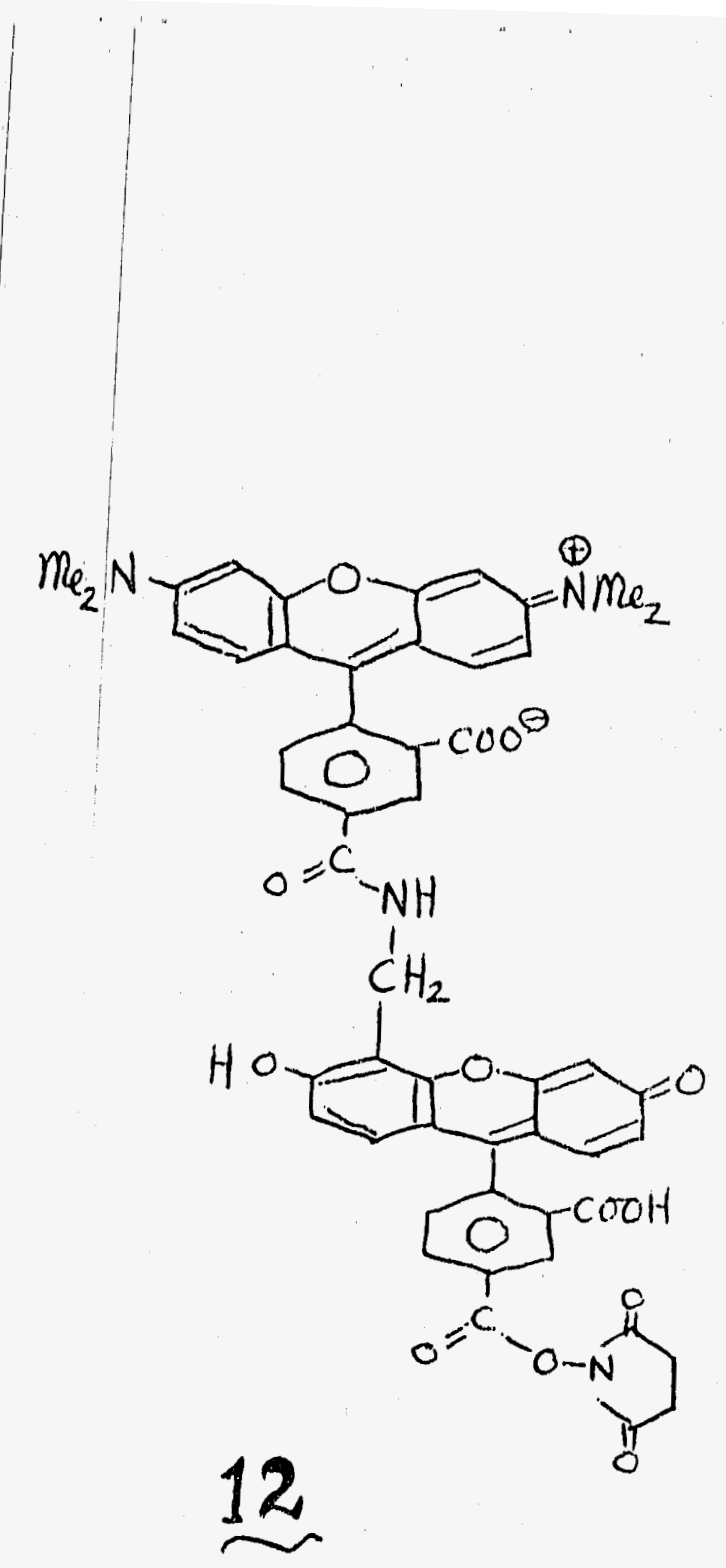

Figure 4 

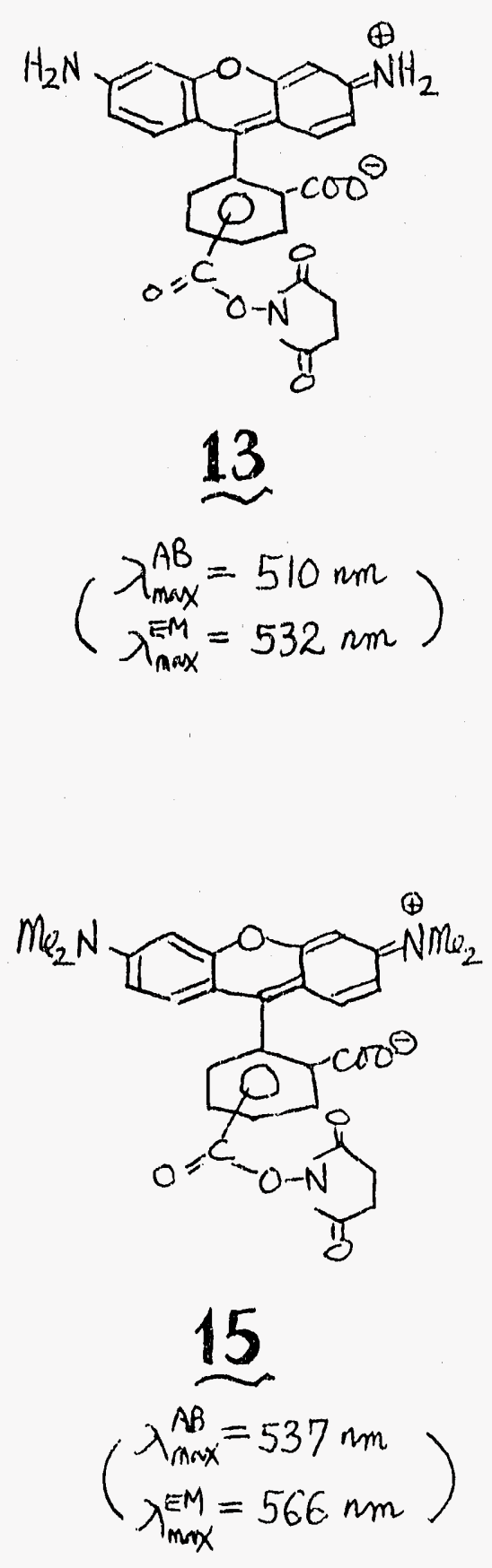
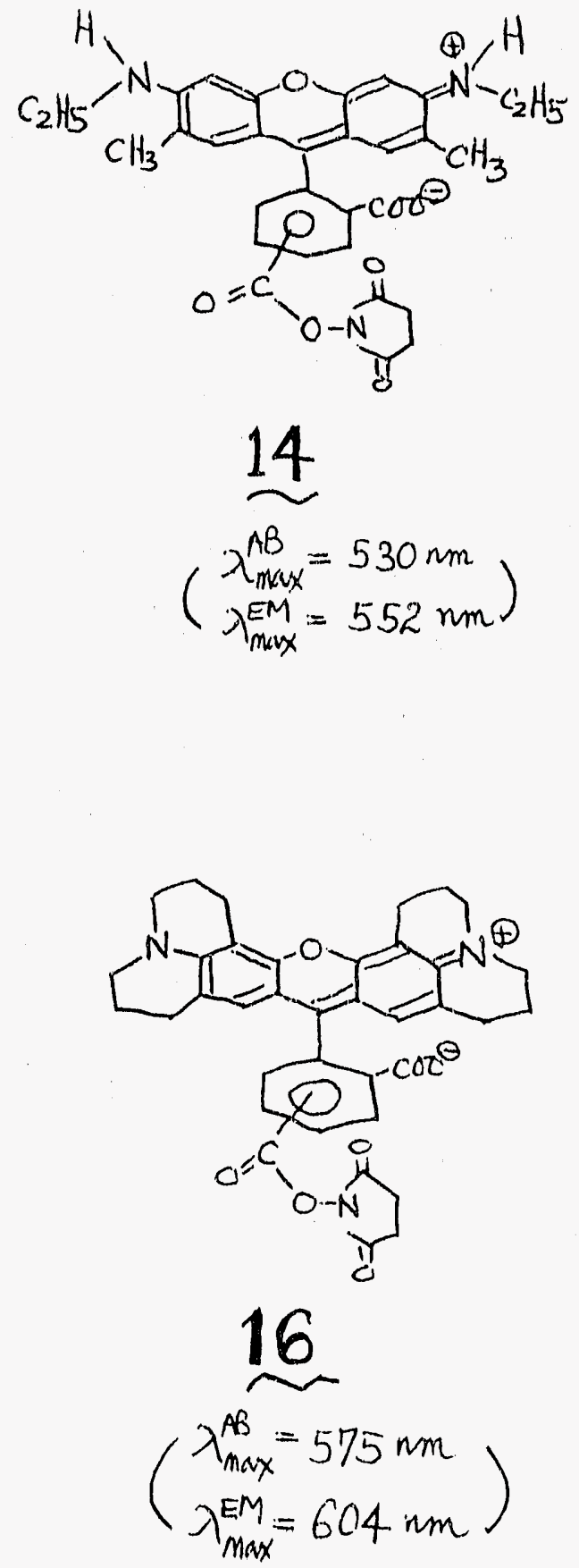

Figure 5 
<smiles>Nc1ccc2oc3c(=O)ccc=3c(-c3cccc(C(=O)O)c3)c3ccc(N)c-3c2c1</smiles>

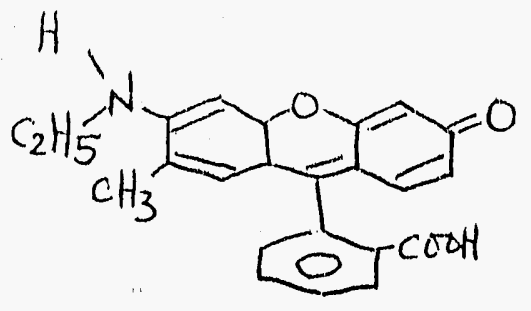

17

18

$\left(\begin{array}{l}\lambda_{\max }^{A B}=492 \mathrm{~mm} \\ \lambda_{\max }^{E M}=517 \mathrm{~mm}\end{array}\right)$

$\left(\begin{array}{l}\lambda_{\max }^{A B}=508 \mathrm{~mm} \\ \lambda_{\max }^{E M}=533 \mathrm{~mm}\end{array}\right)$<smiles>[R14]N([14CH3])c1ccc(C(=C2C=CC(=O)C=C2Oc2ccccc2C(=O)O)c2ccccc2C(=O)O)cc1</smiles>

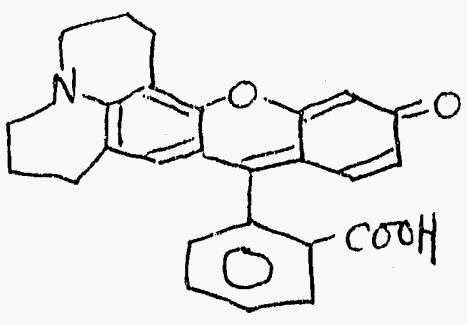

19

20

$\left(\begin{array}{l}\lambda_{\text {m }}^{A B}=513 \mathrm{~mm} \\ \lambda_{m \times x}^{E M}=540 \mathrm{~mm}\end{array}\right)$

$\left(\begin{array}{l}\lambda_{\max }^{A B}=540 \mathrm{~mm} \\ \lambda_{\min }^{E M}=562 \mathrm{~mm}\end{array}\right)$

Figure 6 
<smiles>Cc1c(C)c2c3c(C)cc(C)n3c([PH](F)(F)F)c(C)n2c1C(=O)O</smiles>

21

$\left(\begin{array}{l}\lambda_{\max }^{A B}=512 \mathrm{~mm} \\ \lambda_{\text {Max }}^{E M}=522 \mathrm{~mm}\end{array}\right)$

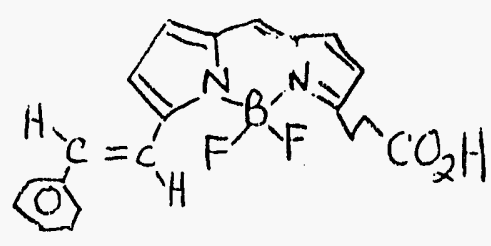

24

$\left(\begin{array}{l}\lambda_{\text {max }}^{A B}=564 \mathrm{~mm} \\ \lambda_{m y x}^{m}=570 \mathrm{~mm}\end{array}\right)$

Figure 7

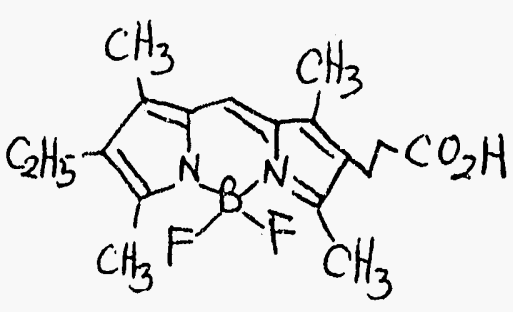

22

$\left(\begin{array}{l}\lambda_{\text {max }}^{A B}=524 \mathrm{~nm} \\ \lambda_{m a x}^{E M}=534 \mathrm{~mm}\end{array}\right)$

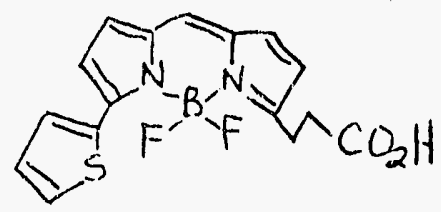

25

$\left(\begin{array}{l}\lambda_{m=x}^{A B}=559 \mathrm{~mm} \\ \lambda_{n \rightarrow x}^{n=x}=568 \mathrm{~mm}\end{array}\right)$

$\left(\begin{array}{l}\lambda_{m x}^{A B}=609 \mathrm{~mm} \\ \lambda_{\text {max }}^{A M}=637 \mathrm{~mm}\end{array}\right)$

23

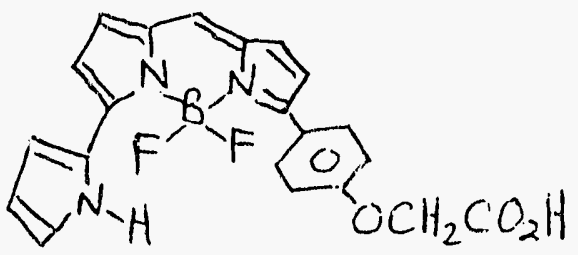

26

$\left(\begin{array}{l}\lambda_{m_{m x}}^{A B}=535 \mathrm{~mm} \\ \lambda_{\max }^{M M}=545 \mathrm{~mm}\end{array}\right)$

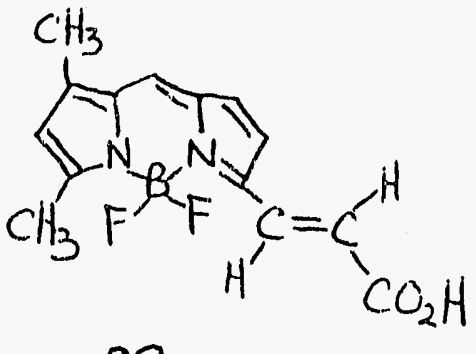



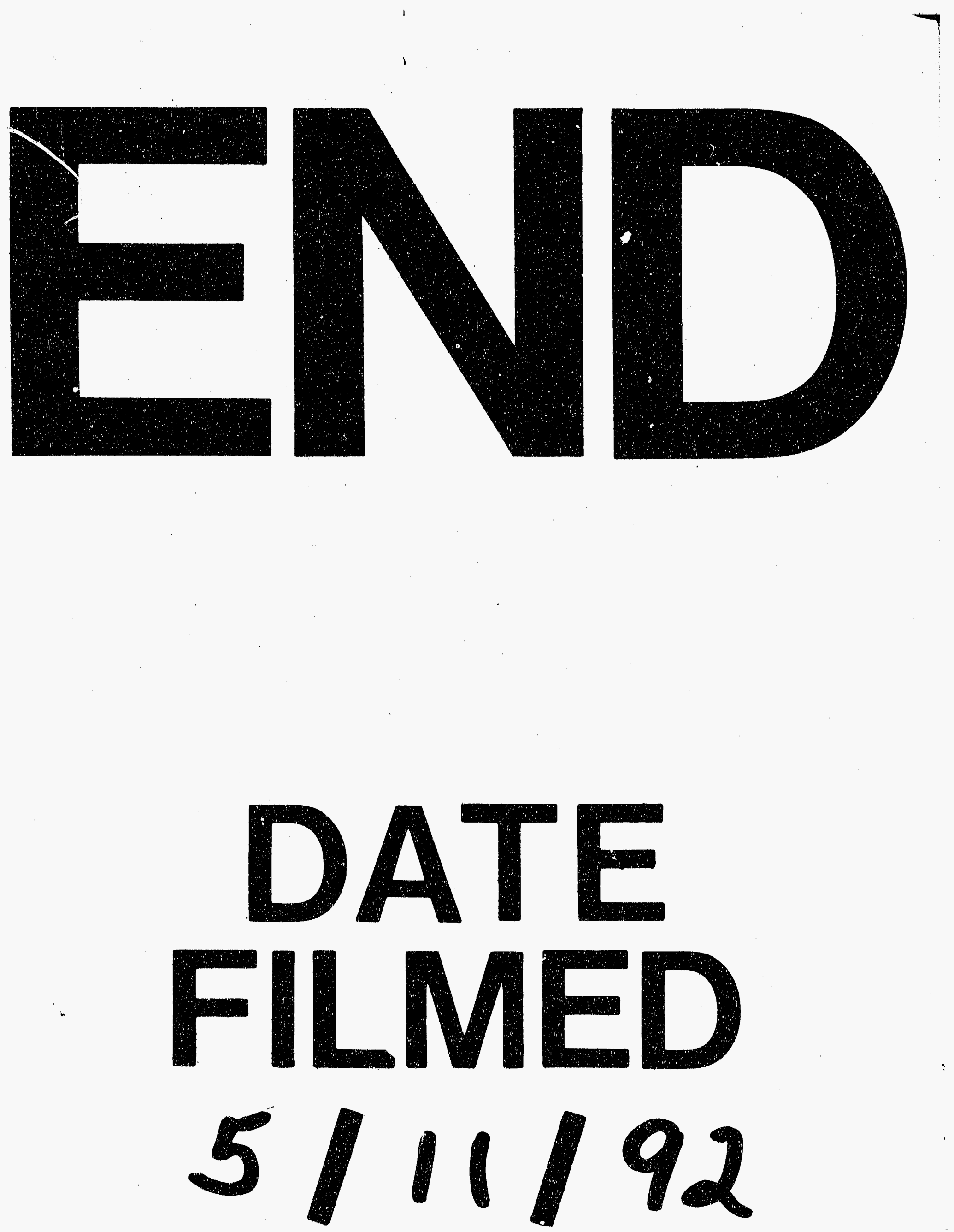

$\mathbf{A}$
$=$ 
